\title{
The effects of a lutein-based supplement on objective and subjective measures of retinal and visual function in eyes with age-related maculopathy - a randomised controlled trial
}

\author{
Emma J. Berrow $^{1,2 *}$, Hannah E. Bartlett ${ }^{1}$, Frank Eperjesi ${ }^{1}$ and Jonathan M. Gibson ${ }^{1,2}$ \\ ${ }^{1}$ Ophthalmic Research Group, School of Life and Health Sciences, Aston University, Birmingham, UK \\ ${ }^{2}$ Heart of England NHS Trust, Birmingham, UK \\ (Submitted 29 March 2012 - Final revision received 9 August 2012 - Accepted 9 August 2012 - First published online 23 October 2012)
}

\begin{abstract}
Lutein and zeaxanthin are lipid-soluble antioxidants found within the macula region of the retina. Links have been suggested between increased levels of these carotenoids and reduced risk for age-related macular disease (ARMD). Therefore, the effect of lutein-based supplementation on retinal and visual function in people with early stages of ARMD (age-related maculopathy, ARM) was assessed using multifocal electroretinography (mfERG), contrast sensitivity and distance visual acuity. A total of fourteen participants were randomly allocated to either receive a lutein-based oral supplement (treated group) or no supplement (non-treated group). There were eight participants aged between 56 and 81 years (65.50 (SD 9.27) years) in the treated group and six participants aged between 61 and 83 years (69.67 (SD 7.52) years) in the non-treated group. Sample sizes provided $80 \%$ power at the $5 \%$ significance level. Participants attended for three visits $(0,20$ and 40 weeks). At 60 weeks, the treated group attended a fourth visit following 20 weeks of supplement withdrawal. No changes were seen between the treated and non-treated groups during supplementation. Although not clinically significant, mfERG ring 3 N2 latency $(P=0.041)$ and ring $4 \mathrm{P} 1$ latency $(P=0.016)$ increased, and a trend for reduction of mfERG amplitudes was observed in rings 1,3 and 4 on supplement withdrawal. The statistically significant increase in mfERG latencies and the trend for reduced mfERG amplitudes on withdrawal are encouraging and may suggest a potentially beneficial effect of lutein-based supplementation in ARM-affected eyes.
\end{abstract}

Key words: Lutein: Macular degeneration: Electroretinography: Electrophysiology

Age-related macular disease (ARMD) is the leading cause of visual loss in industrialised countries ${ }^{(1-3)}$. Present treatments are limited and costly ${ }^{(4,5)}$. The psychological impact ${ }^{(6,7)}$ and reduced quality of life ${ }^{(8,9)}$ that occur during the later stages of the disease are devastating to individuals. As life expectancy increases, ARMD will become more prevalent ${ }^{(1,10,11)}$, highlighting the importance of prevention strategies.

Oxidative stress is thought to increase the risk of ARMD development ${ }^{(12)}$. Thus, the role of antioxidant supplementation is of interest. The carotenoids lutein and zeaxanthin, collectively termed macular pigments, are lipid-soluble antioxidants found within the macula. Links have been suggested between increased levels of these carotenoids and reduced risk for ARMD development ${ }^{(13-15)}$. High-dose antioxidant vitamins $\mathrm{C}, \mathrm{E}, \boldsymbol{\beta}$-carotene and $\mathrm{Zn}$ have also been found to be effective in lowering the OR of developing advanced ARMD in a large trial undertaken by the Age-Related Eye Disease Study ${ }^{(16)}$. High levels of $n-3$ fatty acid consumption $(>75$ percentile) have shown protective effects against the development of advanced ARMD ${ }^{(17)}$.

Cone axons contain the highest amount of macular pigments $^{(18)}$ where tubulin, a protein located in cone photoreceptor cytoskeletons, selectively binds to lutein and zeaxanthin $^{(19)}$. Lutein and zeaxanthin are thought to reduce oxidative damage by filtering short-wavelength blue light within the macula ${ }^{(18)}$ and by quenching light-induced singlet oxygen and related free radicals ${ }^{(20)}$. More recently, antiinflammatory effects of lutein in vitro, in vivo and directly within the retina have been reported ${ }^{(21)}$, suggesting that lutein may reduce the activation of inflammatory pathways in ARMD $^{(22)}$.

Vision can be assessed in numerous ways, as it is composed of many simultaneous functions allowing the perception of form, colour and movement. In most ARMD studies, visual acuity (VA) is used as a measure of visual function. However, this assesses only one small area of the retina, relies on

Abbreviations: AMD, age-related macular degeneration; ARM, age-related maculopathy; ARMD, age-related macular disease; CARMIS, Carotenoids and Antioxidants in Age-related Maculopathy Italian Study; CS, contrast sensitivity; LogMAR, logarithm of the minimum angle of resolution; mfERG, multifocal electroretinogram; VA, visual acuity.

*Corresponding author: E. J. Berrow, email berrowej@aston.ac.uk 
subjective responses and does not provide an overall measure of macular function. Combined objective and subjective testing may provide greater insight into visual and retinal function when diagnosing ARMD and assessing treatment outcomes. Improvements in visual function have been reported in eyes with ARMD in studies involving carotenoids ${ }^{(23-26)}$.

The multifocal electroretinogram (mfERG) objectively assesses localised areas of retinal function ${ }^{(27)}$ by recording electrical potentials from many areas of the retina simultaneously ${ }^{(28)}$, which is useful for identifying focal pathology and monitoring the effects of clinical intervention ${ }^{(29)}$. The mfERG largely assesses cone photoreceptor and bipolar cell function $^{(30)}$, and because macular pigments selectively bind to tubulin within cone photoreceptor axons ${ }^{(19)}$, the aim of the present study was to assess whether a lutein-based nutritional supplement would enhance mfERG measures (the primary outcome measure) in eyes with the earliest form of ARMD, age-related maculopathy (ARM), in concordance with the Carotenoids and Antioxidants in Age-related Maculopathy Italian Study (CARMIS) investigators who found increased central mfERG amplitudes in ARM eyes with lutein-based supplementation $^{(25)}$. Secondary subjective measures assessed were VA and contrast sensitivity (CS).

\section{Subjects and methods}

The research was approved by the Aston University Human Sciences Ethical Committee. The tenets of the Declaration of Helsinki ${ }^{(31)}$ and the consolidated standards of reporting trials $^{(32)}$ were followed. The study was registered with an International Standard Randomised Controlled Trial Number (17842302). Participants were recruited from volunteers at the Aston University eye clinic.

A total of fourteen participants with ARM were randomly allocated, using Microsoft Excel random number generator, to either receive a lutein-based oral supplement (treated group) or no supplement (non-treated group) at visit one. These were from an original cohort of sixteen participants, two of which withdrew without giving reason. Only one eye from each participant was studied. There were eight participants aged between 56 and 81 years (65.50 (SD 9.27) years) in the treated group and six participants aged between 61 and 83 years $(69.67$ (SD 7.52) years) in the non-treated group. All participants attended for three visits: visit one at baseline, visit two at 20 weeks and visit three at 40 weeks. The study was single-masked. At 40 weeks the unmasking occurred, and those in the treated group stopped taking the supplement. At 20 weeks after the treated group had ceased supplementation, all outcome measures were assessed once more for this group (60 weeks).

The supplement name was Ocuvite Duo (Bausch and Lomb). All of the nutrients were within the safe upper levels, as defined by the Food Standards Agency (see Table 1) ${ }^{\text {(33). }}$.

\section{Inclusion criteria}

Suitability for inclusion was evaluated by questionnaire, fundus photographs and VA. For inclusion, participants had
Table 1. Supplement composition

\begin{tabular}{lll}
\hline Ingredients and chemical formula & Safe upper levels/d & \\
\hline Vitamin $\mathrm{C}$ & Dosages \\
$\quad$ (ascorbic acid) $-\mathrm{C}_{6} \mathrm{H}_{8} \mathrm{O}_{6}$ & $\begin{array}{c}1000 \mathrm{mg} \\
\text { (guidance only) }\end{array}$ & $150 \mathrm{mg}$ \\
$\mathrm{Cu}$ (cupric oxide) $-\mathrm{CuO}$ & $10 \mathrm{mg}$ & $400 \mu \mathrm{g}$ \\
Vitamin E & $540 \mathrm{mg}$ & $15 \mathrm{mg}$ \\
$\quad\left(\mathrm{DL}-\alpha\right.$-tocopherol) $-\mathrm{C}_{29} \mathrm{H}_{50} \mathrm{O}_{2}$ & & \\
$\mathrm{Zn}(\mathrm{zinc}$ oxide) $-\mathrm{ZnO}$ & $25 \mathrm{mg}$ & $20 \mathrm{mg}$ \\
Lutein $-\mathrm{C}_{40} \mathrm{H}_{56} \mathrm{O}_{2}$ & Non-established & $12 \mathrm{mg}$ \\
Zeaxanthin $-\mathrm{C}_{40} \mathrm{H}_{56} \mathrm{O}_{2}$ & Non-established & $0.6 \mathrm{mg}$ \\
$n-3 \mathrm{Consisting} \mathrm{of:}$ & Non-established & $1080 \mathrm{mg}$ \\
EPA $-\mathrm{C}_{20} \mathrm{H}_{30} \mathrm{O}_{2}$ & & $240 \mathrm{mg}$ \\
DHA $-\mathrm{C}_{22} \mathrm{H}_{32} \mathrm{O}_{2}$ & & $840 \mathrm{mg}$ \\
\hline
\end{tabular}

to provide written informed consent and were required to have: best-corrected distance VA of 0.2 logarithm of the minimum angle of resolution (LogMAR) or better (for good mfERG central fixation); clear optical media, as determined by a clear view of the fundus; and no signs of other retinal or optic nerve disease other than ARM (as determined by fundal photography and questionnaire) in the study eye, as well as good general health (as determined by health questionnaire) and no prescribed medication that can affect the retina (as determined by health questionnaire).

The International ARM Group has defined an international classification system for quantifying and defining the different subgroups of ARMD in an attempt to permit easier comparison of research findings between groups ${ }^{(34)}$. They define ARM as a disorder of the macular area, most apparent after the age of 50 years and characterised by areas of soft drusen, hyperpigmentation in the outer retina or choroid with drusen or hypopigmentation of the retinal pigment epithelium with drusen. This classification system was used to recruit ARM participants for the present study.

\section{Exclusion criteria}

Certain factors excluded potential participants, and these were: moderate-to-dense lens opacities, intraocular lens, corneal opacities, glaucoma or ocular hypertension, previous history of intraocular inflammation, previous history of retinal detachment, retinal disease (other than ARM), previous retinal laser, diabetes, systemic hypertension, history of ocular trauma, neurological disease, age-related macular degeneration (AMD) in the studied eye, drugs causing retinal toxicity, previous ocular surgery and epilepsy.

\section{Multi-focal electroretinography}

A VERIS science 6.1 (Electrodiagnostic Imaging) was used to record the mfERG. The stimulus, consisting of sixty-one scaled hexagons, was displayed on a high-resolution, blackand-white cathode ray tube monitor $30 \mathrm{~cm} \times 30 \mathrm{~cm}$, with a frame rate of $75 \mathrm{~Hz}$. The stimulus radius subtended approximately $20^{\circ}$ of the visual field. Each hexagon was independently alternated between white $\left(200 \mathrm{~cd} / \mathrm{m}^{2}\right)$ and black $\left(1 \mathrm{~cd} / \mathrm{m}^{2}\right)$ according to a pseudorandom binary $\mathrm{m}$-sequence ${ }^{(35)}$. Recording time was divided into eight $30 \mathrm{~s}$ segments. Fixation target 


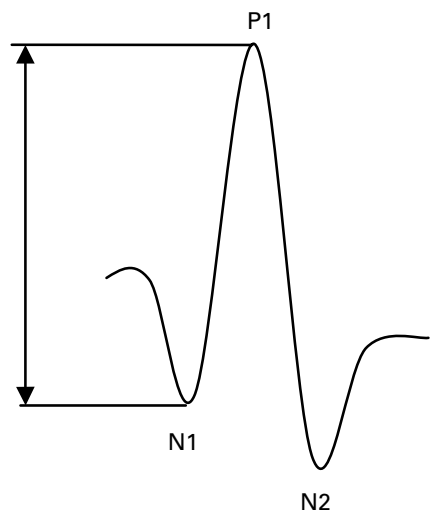

Fig. 1. A normal multifocal electroretinogram response showing N1, P1 and N2 latency. The double-ended arrow demonstrates N1P1 amplitude (source - authors' own drawing).

perception was confirmed before the testing commenced. Each subject's vision was corrected with the VERIS refractor/ camera system (Electrodiagnostic Imaging). The participant's eye was monitored throughout testing using this system. Pupils were dilated with tropicamide 1\% (Bausch and Lomb). Gold cup electrodes filled with Signa gel (Parker Laboratories) were applied to the forehead at $1 \mathrm{~cm}$ posterior to the temporal canthus after cleaning with Nuprep (Weaver and Company). A Dawson Trick Litzkow fibre electrode was placed along the sclera adjacent to the lower eyelid. Proxymetacaine hydrochloride $0.5 \%$ (Bausch and Lomb) was instilled to minimise blinking during recording. Any recordings contaminated with artefact were repeated. The untested eye was obscured throughout recordings. One iteration of artefact removal was performed for each mfERG recording, removing small eye movement artefacts. The mfERG measures were N1, P1 and N2 latency and N1P1 amplitude (Fig. 1), and these were assessed for five rings of retinal eccentricity (Fig. 2).

\section{Contrast sensitivity}

The Pelli-Robson CS test (Clement Clarke International Limited) was measured at a $1 \mathrm{~m}$ distance in the same room for each visit, with a background luminance of 142 lux.

\section{Visual acuity}

LOgMAR distance VA testing was measured using a $3 \mathrm{~m}$ ETDRS 750 lux retro-illuminated chart (Sussex Vision). The eye with the best-corrected distance VA was determined at the participant's first visit and this eye was assessed for subsequent visits. If one eye had ARM, this eye was used. If both eyes had ARM, the eye with the best-corrected distance VA was used to ensure good mfERG fixation.

\section{Intraocular pressure}

Non-contact intraocular pressure readings (Topcon CT-80 non-contact tonometer, Topcon) were taken prior to instillation of tropicamide $1 \%$.

\section{Fundus photography}

Central $45^{\circ}$ fundal photographs were taken with a Topcon TRC-NW8 (Topcon) at each visit to determine changes in fundus or media opacity. A central fixation target for each visit was used to ensure identical fundus positioning. No participants progressed from ARM to AMD throughout the trial.

\section{Food diaries}

Food diaries were given at visits one, three and four, and were filled in over two weekdays and one weekend day. Data were analysed using the Weighted Intake Software Package (Tinuviel). Lutein values were taken from the United States Department of Agriculture nutrient databank, release 23 (http://www.ars.usda.gov/SP2UserFiles/Place/12354500/Data/ SR23/nutrlist/sr23a338.pdf). Although not contained within the supplement, dietary levels of carotene (plant forms of vitamin A) and retinol (animal forms of vitamin A) were also analysed because of the potential protective effect of vitamin A against $\operatorname{ARMD}^{(16,36)}$.

\section{Statistical methods}

All sample sizes provided $80 \%$ power at the 5\% significance level for all outcome measures to detect a change in effect size greater than 0.1 LogMAR for VA, 0.30 log units for CS, $66.08 \mathrm{nV} / \mathrm{deg}^{2}$ for central mfERG amplitude and $6.0 \mathrm{~ms}$ for central mfERG latency. These effect sizes were based on previous studies $^{(25,37-39)}$. All statistical analyses were undertaken using SPSS 16.0 (IBM). A $\chi^{2}$ test for independence demonstrated no difference between treated and non-treated groups for sex $\left(\chi^{2}=0.729, P=0.393\right)$. Group baseline characteristics are detailed in Table 2 and were analysed using independentsamples $t$ tests. A mixed between-within subjects ANOVA was conducted to explore the effects of supplementation compared with no treatment for all outcome measures. This provided analysis of the between-subjects variable (treated and

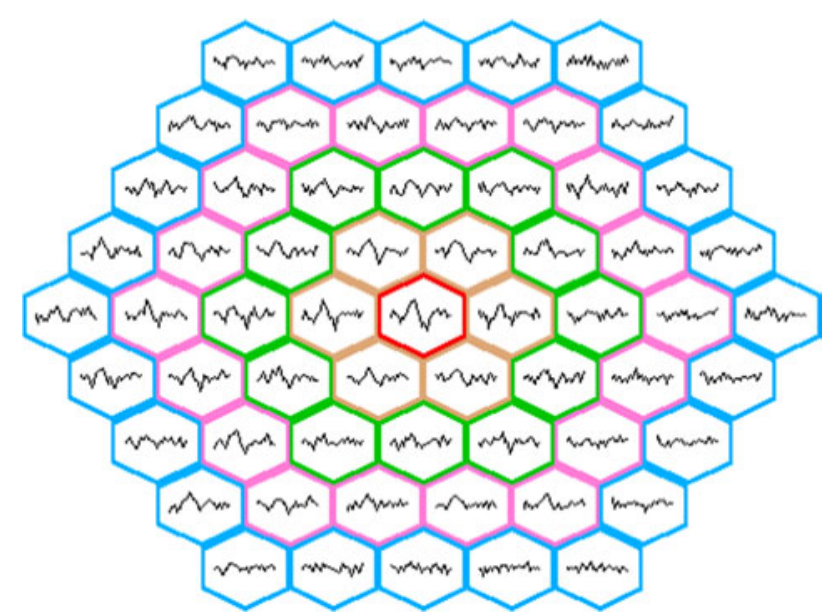

Fig. 2. Grouping of the multifocal electroretinogram areas analysed. Ring 1 - central hexagon (approximately $0.0-2.5^{\circ}$ ), ring 2 (approximately $2.5-5.0^{\circ}$ ) surrounds the central hexagon, ring 3 (approximately $5.0-10.0^{\circ}$ ) surrounds ring 2 , ring 4 (approximately $10.0-15.0^{\circ}$ ) surrounds ring 3 , ring 5 (approximately $15.0-20.0^{\circ}$ ) surrounds ring 4. (A colour version of this figure can be found online at http://www.journals.cambridge.org/bjn). 
Table 2. A summary of baseline characteristics using independent-samples $t$ tests (Mean values and standard deviations)

\begin{tabular}{|c|c|c|c|c|c|c|}
\hline \multirow[b]{2}{*}{ Variable } & \multicolumn{2}{|c|}{ Treated group $(n 8) \dagger$} & \multicolumn{2}{|c|}{ Non-treated group $(n 6) \ddagger$} & \multirow[b]{2}{*}{$t$} & \multirow[b]{2}{*}{$P$} \\
\hline & Mean & SD & Mean & SD & & \\
\hline Age (years) & $65 \cdot 50$ & 9.27 & $69 \cdot 67$ & 7.52 & -0.87 & 0.40 \\
\hline Smoking (pack-years) ${ }^{\star}$ & 7.04 & 9.42 & 13.5 & $15 \cdot 86$ & -0.96 & 0.36 \\
\hline Spherical equivalent (D) & 0.72 & $2 \cdot 27$ & 0.96 & $2 \cdot 30$ & -0.20 & 0.85 \\
\hline Axial length $(\mathrm{mm})$ & $23 \cdot 11$ & 0.86 & 23.48 & 1.25 & -0.65 & 0.53 \\
\hline \multicolumn{7}{|l|}{ Baseline dietary questionnaires } \\
\hline Dietary Cu (mg) & 1.08 & 0.30 & 1.22 & 0.51 & -0.60 & 0.57 \\
\hline Dietary Zn (mg) & 8.07 & 2.43 & 8.98 & 5.06 & -0.41 & 0.69 \\
\hline Dietary retinol $(\mu \mathrm{g})$ & $492 \cdot 00$ & 347.89 & 288.25 & 97.02 & 0.78 & 0.46 \\
\hline Dietary carotene $(\mu \mathrm{g})$ & 2399.71 & $1530 \cdot 05$ & $1807 \cdot 25$ & $882 \cdot 10$ & 0.70 & 0.50 \\
\hline Dietary vitamin $\mathrm{E}(\mathrm{mg})$ & 5.06 & 1.60 & $7 \cdot 21$ & 3.55 & -1.41 & 0.19 \\
\hline Dietary vitamin C (mg) & 98.71 & 49.42 & $112 \cdot 00$ & 59.02 & -0.40 & 0.70 \\
\hline Dietary lutein and zeaxanthin $(\mu \mathrm{g})$ & $1606 \cdot 50$ & $686 \cdot 20$ & $2550 \cdot 02$ & $1557 \cdot 50$ & -1.42 & 0.19 \\
\hline Dietary $n-3(\mathrm{~g})$ & 0.14 & 0.10 & 0.17 & 0.18 & -0.35 & 0.73 \\
\hline
\end{tabular}

${ }^{*}$ Pack-years $=($ cigarettes smoked $/ \mathrm{d} \times$ years smoked $) / 20$. $\dagger n 7$ for baseline dietary questionnaire data. $\ddagger n 4$ for baseline dietary questionnaire data.

non-treated group), within-subject variable (time) and interaction effects on outcome measures. All eight treated participants underwent mfERG at visit one and visit three. Therefore, to exploit this greater sample size, paired-samples $t$ tests were carried out. An independent-samples $t$ test assessed any dietary differences throughout the study. Paired-samples $t$ tests were used to assess the effects of withdrawal of the supplement between visits three and four.

\section{Results}

\section{Supplementation}

Due to technical difficulties, seven participants (four in the treated group and three in the non-treated group) undertook mfERG for all three visits. However, VA and CS were undertaken on all participants. All participants were Caucasian. Of the treated group, seven participants ( $88 \%$ of the eight treated participants) returned their baseline dietary questionnaire. Of the non-treated group four returned their baseline dietary questionnaire $(67 \%$ of the six non-treated participants).

For mfERG outcome measures, there was no significant interaction between the treated and non-treated groups between visits one and three. There were no effects for time or group (treated $v$. non-treated $)$, except for rings $3(P=0 \cdot 021)$ and $4(P=0 \cdot 016)$ N1P1 amplitude for time, with the amplitudes of both rings for both groups reducing between visits one and two and increasing between visits two and three. These results have no clinical significance, as these values are less than the coefficient of repeatability values obtained within our laboratory.

Paired-samples $t$ tests between visits one and three demonstrated that, although not clinically significant, there was statistical significance for ring 3 N1P1 amplitude, with amplitude increasing by $6.67 \mathrm{nV} / \mathrm{deg}^{2}(t=-2.787, P=0.027)$ between visits one and three.

All participants undertook VA and CS assessment at all three visits. There were no significant changes between the treated and non-treated groups over 40 weeks for these measures.
Of the seven people in the treated group who completed the baseline dietary questionnaire, five completed a further questionnaire at visit three. Of the four people in the nontreated group who completed the baseline dietary questionnaire, four completed a further questionnaire at visit three. An independent-samples $t$ test demonstrated no significant difference for any of the dietary components between visits one and three in the treated group or for the non-treated group $(P>0 \cdot 05)$.

Participants were asked to return any boxes of the supplement that were not taken at visit 3. Those who forgot to bring back the tablets were asked to contact an investigator after counting tablets at home. Patient compliance was elicited using supportive language to minimise the number of participants concealing supplement non-adherence ${ }^{(40)}$ and reporting lower levels of remaining tablets than was actually the case. The sole reason for non-adherence was forgetfulness. The mean compliance, measured as the percentage of tablets taken, was $81 \cdot 1$ (SD 13.0)\%.

\section{Withdrawal}

All eight treated participants at visit 3 were able to return for visit 4 and undertook all tests for both visits. Of the eight participants, four completed a dietary questionnaire at visit 3, of which three completed a further questionnaire at visit 4 .

The data were analysed using paired-samples $t$ tests, as parametric assumptions were met. After withdrawal of the nutritional supplement for 20 weeks, no statistically significant changes were seen for any outcome measure except for mfERG ring $3 \mathrm{~N} 2$ latency $(P=0 \cdot 041)$ and ring $4 \mathrm{P} 1$ latency $(P=0.016)$, which increased. There was a trend for reduced mfERG amplitudes in rings 1, 3 and 4, although not clinically significant based on mfERG repeatability studies. There were no changes in dietary levels of nutrients during the withdrawal period. 


\section{Discussion}

There were no changes to outcome measures when comparing treated and non-treated groups over 40 weeks. An increase in mfERG ring 3 N1P1 amplitude was seen over 40 weeks in the treated group alone when paired-samples $t$ tests were used to exploit the greater sample size. This improvement in retinal function may be due to antioxidant and antiinflammatory effects of the nutrients within the supplement, in reducing early, underlying inflammation in the outer retina and photoreceptors ${ }^{(15,21,36,41)}$. Although not clinically significant, based on repeatability studies from our laboratory, on withdrawal of the supplement, there was a trend for a reduction in mfERG amplitudes in rings 1, 3 and 4 and statistically significant increases to mfERG ring 3 N2 latency and ring 4 P1 latency. Again, this may be due to the withdrawal of antioxidant and anti-inflammatory protection provided by the supplement.

Richer concluded that lutein supplementation in fourteen males with atrophic AMD improved CS and $\mathrm{VA}^{(26)}$, although CS and VA assessment, ARMD type and sex composition differed from the Aston study. Supplement formulation was also incompatible, with spinach used for eleven subjects and the remainder receiving a lutein-based antioxidant.

The Lutein Antioxidant Supplementation Trial investigators concluded that 12 months' supplementation with $10 \mathrm{mg}$ lutein, or $10 \mathrm{mg}$ lutein with other carotenoids, antioxidants and minerals, improved $\mathrm{CS}^{(24)}$. A larger sample size of ninety participants, predominantly male, with atrophic AMD means that results may not be comparable with the Aston study.

Bartlett \& Eperjesi ${ }^{(42)}$ found that supplementation with $6 \mathrm{mg}$ of lutein combined with vitamins and minerals was not beneficial for improving $\mathrm{CS}$ in fifteen eyes with ARM or atrophic AMD over 9 months. Although the method of assessing CS was comparable to the Aston study, supplementation formulation differed.

The Taurine, Omega- 3 fatty acids, Zinc, Antioxidant, Lutein study concluded that $76.7 \%$ of the thirty-seven eyes with atrophic AMD had stable or improved VA at 6 months when taking an $8 \mathrm{mg}$ lutein-based supplement. Although VA assessment was the same as for the Aston study, the type of ARMD and supplement composition differed ${ }^{(23)}$.

The CARMIS investigators found that $10 \mathrm{mg}$ of lutein-based supplement over 12 months increased mfERG N1P1 amplitudes in rings 1 and 2 in fifteen eyes with ARM or non-central geographic atrophy ${ }^{(25)}$. As the supplement composition and ARMD categories were not the same as for the present study, it is difficult to compare the CARMIS study with the Aston study. The CARMIS investigators did not report dietary levels of lutein and zeaxanthin throughout the study; thus it is difficult to determine if mfERG changes seen were due to supplementary or dietary changes in lutein and zeaxanthin.

There is paucity in the literature about the effect of lutein supplement withdrawal on measures of visual and retinal function. A study assessing the effects of a lutein-based supplement on the focal electroretinogram noted that a participant who had stopped taking the supplement had reduced focal electroretinogram amplitudes ${ }^{(43)}$. The Aston study is the first study to assess the effects of withdrawal of lutein-based supplementation on mfERG measures in human eyes with ARM.

Although freely available and relatively easy to measure, VA alone is not ideal to determine visual function in ARMD due to its subjective nature. Other measures of visual function need to be developed to better assess subtle changes in disease and the effect of clinical intervention. The introduction of repeatable, objective tests for macular pigments may provide additional accurate information with regard to lutein and zeaxanthin accumulation within the retina, leading to more favourable comparisons with objective measures of retinal function like the mfERG.

\section{Study limitations}

Food diaries were prospective and completed over several days to provide detailed dietary information. Return rates were lower than anticipated. A recall food diary would have provided a greater number of returned questionnaires as participants complete these during visits, although not as accurate due to participants having to remember food intake over a $3 \mathrm{~d}$ period.

The lack of placebo in the non-treated group may be considered a limitation. The time constraints of the trial and the costly and time-consuming nature of placebo manufacture meant that placebo allocation was not feasible. It may be argued that participants not receiving a treatment may bias the results by not engaging in the study as enthusiastically as those given a treatment or placebo. However, the results of the trial did not reflect this.

It may be argued that combining supplement components does not allow for the assessment of individual effects of each active ingredient on measures of visual function. However, many eye supplements available are combination supplements due to the synergistic nature of ingredients. Appropriate examples of synergism include $\mathrm{Cu}$ and $\mathrm{Zn}$ combinations required for $\mathrm{Cu}-\mathrm{Zn}$ superoxide dismutase, a part of the antioxidant system within the retinal pigment epithelium and retina ${ }^{(44)}$, and increased bioavailability of lutein with the addition of certain oils ${ }^{(45,46)}$. Because the causes of ARMD are multi-faceted, it may be that multi-ingredient supplementation is of more benefit than a single nutrient. Indeed, the Age-Related Eye Disease Study investigators found that $\mathrm{Zn}$ and antioxidants reduced the relative risk of developing advanced AMD by 21 and 17\%, respectively. When $\mathrm{Zn}$ and antioxidants were combined, the risk reduced further by $25 \%{ }^{(16)}$. Future studies comparing a lutein-containing and a lutein-absent supplement may better determine the role of lutein effects on visual and retinal function.

In summary, the CARMIS investigators' findings were not replicated in the Aston study. Extending on this, mfERG latency, subjective measures of visual function, dietary levels of lutein and zeaxanthin and supplement withdrawal were also assessed in the Aston study. The present study is the 
first to assess the effect of withdrawal of lutein-based supplementation on mfERG measures in ARM.

\section{Acknowledgements}

The authors would like to thank Bausch and Lomb, KingstonUpon-Thames, Surrey, UK for funding the research position and supplying the Ocuvite Duo nutritional supplement. E. J. B. completed the data collection, performed statistical analysis of the data and wrote the manuscript. H. E. B. and F. E. conceptualised and supervised the research, and edited the manuscript. J. M. G. supervised the research and edited the manuscript. The authors declare no competing financial interests.

\section{References}

1. Yong VK, Morgan WH, Cooper RL, et al. (2006) Trends in registered blindness and its causes over 19 years in Western Australia. Ophthalmic Epidemiol 13, 35-42.

2. Klein R, Klein BEK, Tomany SC, et al. (2002) Ten-year incidence and progression of age-related maculopathy. Ophthalmology 109, 1767-1779.

3. Evans J \& Wormald R (1996) Is the incidence of registrable age-related macular degeneration increasing? Br J Ophthalmol 80, 9-14.

4. Colquitt JL, Jones J, Tan SC, et al. (2008) Ranibizumab and pegaptanib for the treatment of age-related macular degeneration: a systematic review and economic evaluation. Health Technol Assess 12, iii-iv, ix-201.

5. Bandello F, Augustin A, Sahel JA, et al. (2008) Association between visual acuity and medical and non-medical costs in patients with wet age-related macular degeneration in France, Germany and Italy. Drugs Aging 25, 255-268.

6. Williams R, Brody BL \& Thomas R (1998) The psychosocial impact of macular degeneration. Arch Ophthalmol 116, $514-520$.

7. Meyer-Ruesenberg B \& Richard G (2010) New insights into the underestimated impairment of quality of life in agerelated macular degeneration - a review of the literature. Klin Monbl Augenheilkd 227, 646-652.

8. Mitchell J, Wolffsohn J, Woodcock A, et al. (2008) The MacDQoL individualized measure of the impact of macular degeneration on quality of life: reliability and responsiveness. Am J Ophthalmol 146, 447-454.

9. Stein JD, Brown MM, Brown GC, et al. (2003) Quality of life with macular degeneration: perceptions of patients, clinicians, and community members. Br J Ophthalmol 87, 8-12.

10. Njiric S, Misljenovic T, Mikulicic M, et al. (2007) Incidence of age-related macular degeneration in correlation with age, sex and occupation. Coll Antropol 31, 107-110.

11. Korobelnik JF, Moore N, Blin P, et al. (2006) Estimating the yearly number of eyes with treatable neovascular age-related macular degeneration using a direct standardization method and a markov model. Invest Ophthalmol Vis Sci 47, 4270-4276.

12. Beatty S, Koh HH, Henson D, et al. (2000) The role of oxidative stress in the pathogenesis of age-related macular degeneration. Surv Ophthalmol 45, 115-134.

13. Mares-Perlman JA, Fisher AI, Klein R, et al. (2001) Lutein and zeaxanthin in the diet and serum and their relation to agerelated maculopathy in the third national health and nutrition examination survey. Am J Epidemiol 153, 424-432.

14. Seddon JM, Ajani UA, Sperduto RD, et al. (1994) Dietary carotenoids, vitamin $\mathrm{A}, \mathrm{C}$ and $\mathrm{E}$ and advanced age-related macular degeneration. Eye Disease Case-Control Study Group. JAMA 272, 1413-1420.

15. EDCCS Group (1993) Antioxidant status and neovascular age-related macular degeneration. Eye Disease Case-Control Study Group. Arch Ophthalmol 111, 104-109.

16. AREDS Research Group (2001) A randomized, placebocontrolled, clinical trial of high-dose supplementation with vitamins $\mathrm{C}$ and $\mathrm{E}$, beta carotene, and zinc for age-related macular degeneration and vision loss - AREDS Report no. 8. Arch Ophthalmol 119, 1417-1436.

17. Chiu CJ, Klein R, Milton RC, et al. (2009) Does eating particular diets alter the risk of age-related macular degeneration in users of the Age-Related Eye Disease Study supplements? Br J Ophthalmol 93, 1241-1246.

18. Snodderly DM, Auran J \& Delori F (1984) The macular pigment II. Spatial distribution in primate retinas. Invest Ophthalmol Vis Sci 25, 674-685.

19. Bernstein PS, Balashov NA, Tsong ED, et al. (1997) Retinal tubulin binds macular carotenoids. Invest Ophthalmol Vis Sci 38, 167-175.

20. Li B, Ahmed F \& Bernstein PS (2010) Studies on the singlet oxygen scavenging mechanism of human macular pigment. Arch Biochem Biophys 504, 56-60.

21. Kijlstra A, Tian Y, Kelly ER, et al. (2012) Lutein: more than just a filter for blue light. Prog Retin Eye Res 31, 303-315.

22. Kijlstra A, La Heij E \& Hendrikse F (2005) Immunological factors in the pathogenesis and treatment of age-related macular degeneration. Ocul Immunol Inflamm 13, 3-11.

23. Cangemi FE (2007) TOZAL Study: an open case control study of an oral antioxidant and omega-3 supplement for dry AMD. BMC Ophthalmol 7, 3.

24. Richer S, Stiles W, Statkute L, et al. (2004) Double-masked, placebo-controlled, randomized trial of lutein and antioxidant supplementation in the intervention of atrophic age-related macular degeneration: the Veterans LAST study (Lutein Antioxidant Supplementation Trial). Optometry 75, 216-230.

25. Parisi V, Tedeschi M, Gallinaro G, et al. (2008) Carotenoids and antioxidants in age-related maculopathy italian study: multifocal electroretinogram modifications after 1 year. Ophthalmology 115, 324-333.e2.

26. Richer S (1999) ARMD-pilot (case series) environmental intervention data. J Am Optom Assoc 70, 24-36.

27. Hood DC, Odel JG, Chen CS, et al. (2003) The multifocal electroretinogram. J Neuroophthalmol 23, 225-235.

28. Sutter EE \& Tran D (1992) The field topography of ERG components in man. 1. The photopic luminance response. Vision Res 32, 433-446.

29. Berrow EJ, Bartlett HE, Eperjesi F, et al. (2010) The electroretinogram: a useful tool for evaluating age-related macular disease? Doc Ophthalmol 121, 51-62.

30. Hood DC, Frishman LJ, Saszik S, et al. (2002) Retinal origins of the primate multifocal ERG: implications for the human response. Invest Ophthalmol Vis Sci 43, 1673-1685.

31. World Medical Association Inc. (2009) Declaration of Helsinki. Ethical principles for medical research involving human subjects. J Indian Med Assoc 107, 403-405.

32. Schulz K, Altman D, Moher D, et al. (2010) CONSORT 2010 Statement: updated guidelines for reporting parallel group randomised trials. BMC Med $\mathbf{8}, 18$.

33. FSA (2003) Expert Group on Vitamins and Minerals - Safe Upper Levels for Vitamins and Minerals. London: Food Standards Agency.

34. Bird AC, Bressler NM, Bressler SB, et al. (1995) An international classification and grading system for age-related maculopathy and age-related macular degeneration. Surv Ophthalmol 39, 367-374. 
35. Sutter EE (1991) The fast M-transform - a fast computation of cross-correlations with binary M-sequences. SIAM J Comput 20, 686-694.

36. West S, Vitale S, Hallfrisch J, et al. (1994) Are antioxidants or supplements protective for age-related macular degeneration? Arch Ophthalmol 112, 222-227.

37. Lovie-Kitchin JE (1988) Validity and reliability of visual acuity measurements. Ophthalmic Physiol Opt 8, 363-370.

38. Elliott DB, Sanderson K \& Conkey A (1990) The reliability of the Pelli-Robson contrast sensitivity chart. Ophthalmic Physiol Opt 10, 21-24.

39. Dolan F, Sandinha T, Purdy A, et al. (2006) Vitamin A deficiency modifies the mfERG: a case study of rod influence on the mfERG. Doc Ophthalmol 112, 31-34.

40. Hahn SR (2009) Patient-centered communication to assess and enhance patient adherence to glaucoma medication. Ophthalmology 116, S37-S42.

41. Crabtree DV, Adler AJ \& Snodderly DM (1996) Radial distribution of tocopherols in rhesus monkey retina and retinal pigment epithelium-choroid. Invest Ophthalmol Vis Sci37, 61-76.
42. Bartlett HE \& Eperjesi F (2007) Effect of lutein and antioxidant dietary supplementation on contrast sensitivity in age-related macular disease: a randomized controlled trial. Eur J Clin Nutr 61, 1121-1127.

43. Falsini B, Piccardi M, Iarossi G, et al. (2003) Influence of short-term antioxidant supplementation on macular function in age-related maculopathy. A pilot study including electrophysiologic assessment. Ophthalmology 110, 51-60.

44. Erie JC, Good JA, Butz JA, et al. (2009) Reduced zinc and copper in the retinal pigment epithelium and choroid in age-related macular degeneration. Am J Ophthalmol 147, $276-282$.

45. O'Connell O, Ryan L, O'Sullivan L, et al. (2008) Carotenoid micellarization varies greatly between individual and mixed vegetables with or without the addition of fat or fiber. Int J Vitam Nutr Res 78, 238-246.

46. Lakshminarayana $\mathrm{R}$, Raju M, Keshava Prakash MN, et al. (2009) Phospholipid, oleic acid micelles and dietary olive oil influence the lutein absorption and activity of antioxidant enzymes in rats. Lipids $\mathbf{4 4}, 799-806$. 